\title{
An Electrochemical Outlook on Tamoxifen Biotransformation: Current and Future Prospects
}

\author{
Jorge M.P.J. Garrido ${ }^{1,2^{*}}$, E. Manuela P.J. Garrido ${ }^{1,2}$, Ana Maria Oliveira-Brett ${ }^{3}$, Fernanda Borges ${ }^{2 *}$ \\ ${ }^{1}$ Departamento de Engenharia Química, Instituto Superior de Engenharia do Porto, IPP, 4200-072 Porto, Portugal, \\ ${ }^{2}$ CIQ/Departamento de Química e Bioquímica, Faculdade de Ciências, Universidade do Porto, 4169-007 Porto, Portugal, \\ ${ }^{3}$ Departamento de Química, Faculdade de Ciências e Tecnologia, Universidade de Coimbra, 3001-401 Coimbra, Portugal
}

\begin{abstract}
Tamoxifen is a nonsteroidal antiestrogen that is currently and widely used in the treatment of breast cancer in all of its stages, in adjuvant therapy as a long-term suppressant of tumor recurrence and also as a chemopreventive agent in women that are in high risk of developing this type of estrogen-dependent cancer.

From a toxicological and (bio)analytical point of view the knowledge of the metabolic pathways of a drug is found to be extremely im- portant. So, in the present work the most important tamoxifen biotransformation steps were reviewed in the light of recent pharmacologi- cal data. This overview also includes the current controversy concerning tamoxifen DNA-damaging (genotoxic) versus non-genotoxic mechanisms.

A special focus will be given to the putative application of electrochemical methods as a modern and reliable analytical tool for determi- nation of tamoxifen and its metabolites. Moreover, the potential of DNA electrochemical sensors for detection of structural damage to DNA as a basis for toxicity screening is highlighted.
\end{abstract}

Future prospects looking for the importance of developing new analytical methodologies are also discussed.

Keywords: Biotransformation, breast cancer, DNA, electrochemistry, sensor, Tamoxifen.

\section{INTRODUCTION}

Selective estrogen receptor modulators (SERMs), known previously as "antiestrogens", are therapeutic agents used for the prevention and treatment of several diseases such as osteoporosis and breast cancer. Currently, there are two classes of clinically approved SERMs the triphenylethylene (e.g., tamoxifen) and the benzothiophene derivatives (e.g., raloxifene) [1-3]. Despite the development of newer SERMs, namely toremifene, tamoxifen still remains the SERM of first choice for the treatment of hormoneresponsive breast cancer

Tamoxifen (TAM, Fig. 1), [Z-1-[4-(2-dimethylamino)ethoxy] phenyl-1,2-diphenylbut-1-ene], the class representative of triphenylethylenes, is a SERM that has been used for nearly three decades as an adjuvant and/or chemotherapeutic agent for the treatment of all stages of hormone-dependent breast cancer [4-6].

Tamoxifen has the greatest therapeutic effect on estrogen receptor (ER) positive tumors, but women with both estrogen receptor positive and negative tumors show an objective remission rate of up to $30 \%$ and an improved life expectancy $[3,5,6]$.

TAM was first used in 1971 as an antineoplastic and was approved for use by the Food and Drug Administration (FDA) in 1977 for the treatment of advanced breast cancer in women. In 1985, FDA approved the use of tamoxifen as an adjuvant therapy in postmenopausal women with node-positive breast cancer, and in 1986, approval was obtained for its use alone. In 1989, the use of tamoxifen in the treatment of premenopausal women with ERpositive advanced breast cancer was approved by FDA. In 1990, an indication as an adjuvant was approved for pre and postmenopausal patients who had node-positive ER-positive breast cancer. In 1993, the same agency approved the indication for tamoxifen use in the treatment of advanced breast cancer in men [4, 7-10].

More recently, data from a large-scale clinical trial in the United States have shown that the administration of tamoxifen to women at a high risk of developing the disease resulted in a $50 \%$ reduction in the occurrence of invasive breast cancer [11]. This observation has prompted the US FDA to approve tamoxifen use as a prophylactic breast cancer agent in high-risk women.

The optimum duration of the tamoxifen treatment has not been clearly established, but the data so far collected suggest that a 2 year period is not sufficient and at least 5 years are required to be beneficial. In fact tamoxifen administration for 5 years is believed to reduce the risk of recurrence of breast cancer both locally and systemically [10-12].

In addition to antitumour activity, tamoxifen exerts beneficial effects on osteoporosis as shown by clinical trials on long-term treatment of postmenopausal women. It is also described that tamoxifen reduces the incidence of cardiovascular diseases and lowers circulating serum cholesterol $[4,13]$.

\section{TAMOXIFEN MECHANISM OF ACTION}

Breast cancer was first recognized to be an estrogen-dependent disease being $75 \%$ of breast cancers positive for ER. The stimulation of estrogen receptor by estradiol is often associated with the development of breast cancer and in turn one of the principal treatment strategies involves estrogen withdrawal [3-5]. As a selective estrogen receptor modulator tamoxifen competes with estrogen for binding to the estrogen receptor and therefore inhibits tumor growth by interfering with the survival and proliferative signals regulated by estrogen [6]. Tamoxifen can act either as an estrogen-antagonist or as an estrogen-agonist depending on the tissue in which operates $[4,6]$.

In breast tissue, tamoxifen mainly act as an estrogen antagonist stopping or reducing estrogen dependent cancer cell proliferation even though it can operate as an estrogen agonist on other parts of the body. In fact, in postmenopausal women tamoxifen exerts estrogenic activity in the endometrium, vaginal epithelium, bone and 



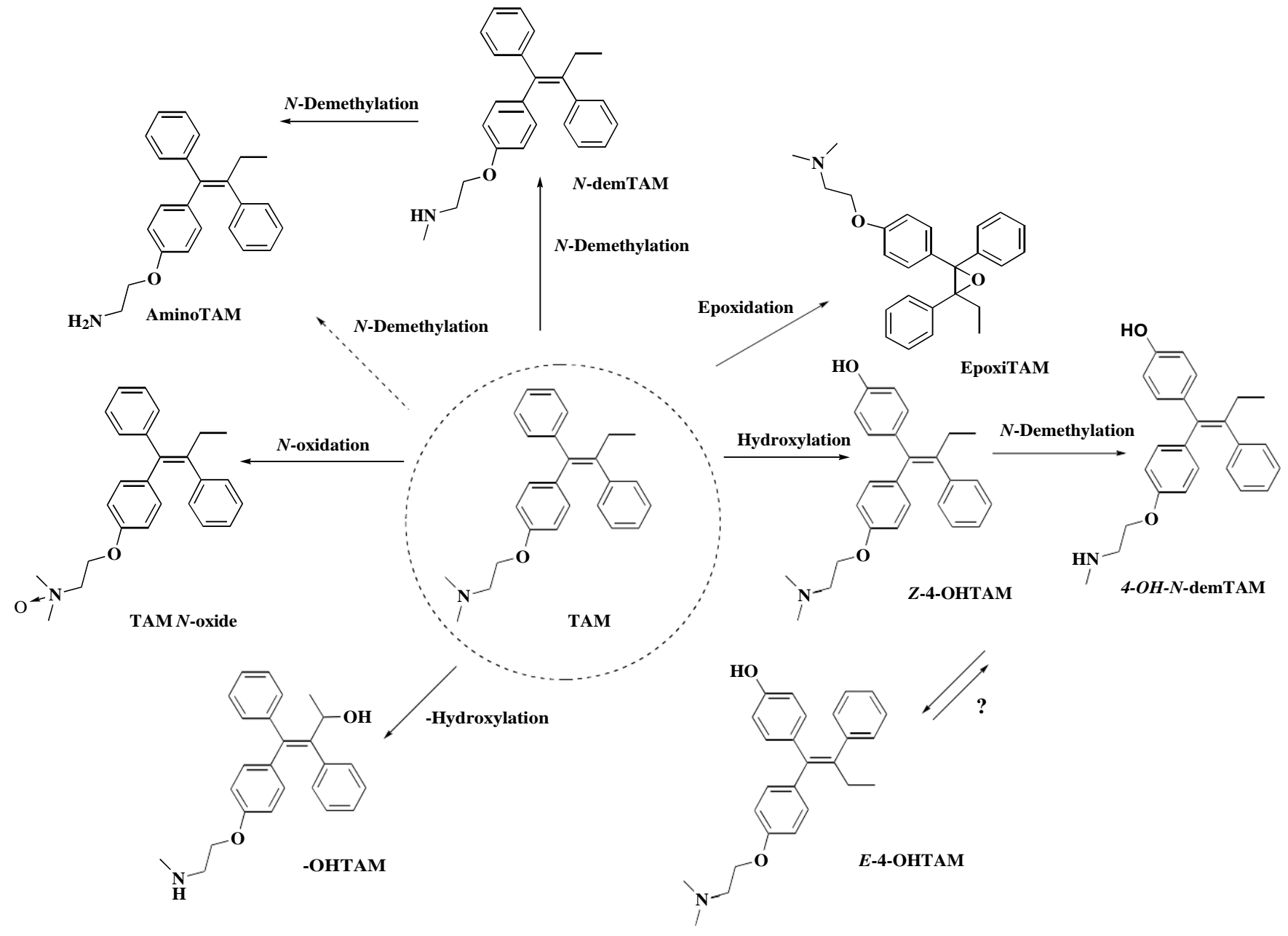

Fig. (1). Tamoxifen (TAM) and its main phase I metabolites.

on serum lipids $[3,4,6]$. The overall benefits of the described activity are connected with protective effects against cardiovascular disease and osteoporosis in breast cancer patients [5].

In addition it was also described a tamoxifen binding effect to the microsomal antiestrogen binding site, interaction with protein kinase C, calmodulin-dependent enzymes and acyl coenzyme A: cholesterol acyl transferase. It is believed that each of these tamoxifen targets might explain some of its genomic as well as nongenomic effects [8-10].

In summary, one can assume that tamoxifen mechanism is still a complex subject matter, being its action both species and tissue specific, and potentially dependent on the duration of use. Accordingly, the current breast cancer therapeutic strategy involves the blocking of the estrogen action on tumor cells via different approaches: a) preventing the binding of estrogen to its receptor by using an antiestrogen compound; b) preventing the biosynthesis of estrogen through the use of aromatase inhibitor; or c) downregulating estrogen receptor protein levels using a pure antiestrogen $[9,10]$.

\section{BENEFITS VS RISKS OF TAMOXIFEN}

Because of tamoxifen pro-estrogenic action on other target tissue sites, its use is often associated with various beneficial effects. In fact it appears to maintain or restore bone mineral density and reduce cholesterol levels in women being treated for breast cancer [5, 10-13].
Despite tamoxifen therapeutic value, several adverse effects have been recognized related with its use, either as a chemotherapeutic or as a chemopreventive agent, against breast cancer. Among them, the development of uterine and endometrial cancer and venous thrombotic events are the most critical. A serious limitation of tamoxifen therapy is the inevitable appearance of resistance, which occur either de novo or is acquired after several months of treatment $[4,6,12,13]$. Tamoxifen resistance is considered to be related with interindividual variability and genetic factors [11-15].

All the above mentioned aspects are up-and-coming topics on this area and give reason for the need to further explore the potential carcinogenic mechanisms of tamoxifen.

\section{TAMOXIFEN METABOLISM}

Although the adverse effects of tamoxifen have been attributed to its hormonal properties, there is currently a deep interest in the study of TAM biotransformation and identification of metabolites since they could act as chemical carcinogens.

After oral administration, TAM is extensively metabolized by phase I and phase II enzymes into several types of metabolites. In women several phase I TAM metabolites have been characterized, mainly obtained by $N$-demethylation, $N$-oxidation, epoxidation, hydroxylation and isomerization bioactivation reactions $(\mathrm{N}$ demTAM, AminoTAM, TAM $N$-oxide, EpoxiTAM, 4-OHTAM, 4$\mathrm{OH}-\mathrm{N}$-demTAM, -OHTAM and E-4-OHTAM) (Fig. 1) $[2,3,8]$. Demethylation of the aminoethoxy side chain to $N$-demTAM ap- 
pears to be the main route of TAM metabolism [4, 16, 17]. The progressive demethylation of the TAM side chain first to $N$ demTAM, the principal metabolite in humans, and then to AminoTAM does not affect the biological action of tamoxifen [17].

A variety of cytochrome P450 isoforms have been already shown to be involved in phase I metabolic pathways. Recently, it was demonstrated that in humans the 4-hydroxylation of TAM is primarily catalyzed by the hepatic CYP2D6 while the $\mathrm{N}$ demethylation process is catalyzed by CYP3A (Fig. 2) [17]. 4OHTAM metabolite has been shown to possess a high affinity for ERs and more potency than TAM in suppressing estrogendependent cell proliferation. It has been also shown that 4-OH- $N$ demTAM (also known as endoxifen) have a potency similar to 4OHTAM with respect to ER binding affinity, suppression of estrogen-dependent cell growth, and gene expression [4]. Since the plasma concentration of this metabolite in breast cancer patients has been reported to be much higher (over 6-fold) than that of TAM it is believed that it can play also an important role in vivo $[16,17]$.

The formation of various -OHTAM metabolites, obtained by hydroxylation of the -carbon, derived from phase I metabolites ( OHTAM, Fig. 3) has been also reported [11, 18]. These compounds were detected at low concentrations in the plasma of women receiving tamoxifen therapy. -OHTAM $\mathrm{N}$-oxide and -OH- $\mathrm{N}$-demTAM have also been identified as metabolites in a rat liver microsomal system and in the urine of breast cancer patients treated with the drug. All the mentioned -OHTAM metabolites has been reported to be a substrate of hydroxysteroid(alcohol) sulfotransferases, enzymes involved in its subsequent activation, even though this step has not been systematically studied [4]. Human phenol-sulfating and estrogen sulfotransferases have been proposed as the major enzymes involved in the sulfation of 4-OHTAM $[18,19]$.

Tamoxifen metabolism has been shown to generate, either chemically or enzymatically, at least three different electrophilic metabolites that include a carbocation, a quinone methide and $o$ quinone (Fig. 4) [11, 16]. These highly reactive species can undergo subsequent processes of polymerization with themselves and/or with biomacromolecules and endogenous antioxidants (Fig. 5 and Fig. 6) $[20,22]$. The $o$-quinone resulting from oxidation of the catechol metabolite, 3,4-diOHTAM, can alkylate amino acid residues on proteins, even though the functional significance of the binding of active metabolites of tamoxifen to proteins is still unclear. The $o$-quinone can cause damage to cellular DNA through generation of reactive oxygen species, and/or alkylation of DNA [4, 21-23]. There is now good evidence that -OHTAM plays an important role in the formation of the electrophilic species responsible for tamoxifen-mediated DNA and protein damage (Fig. 1 and Fig. 7). The carbocation species can undergo a facile rotation around the central double bond and therefore TAM-DNA adducts can exist, due to the potential for isomerization, as four diastereoisomers. Nevertheless the E forms of these adducts predominate [20-23]. Scarce studies have addressed this topic and consequently many conjugates/adducts were never detected in biological fluids because they have never been specifically looked for yet.

It has been previously shown that -hydroxytamoxifen undergo a phase II pathway and is excreted as -OHTAM- $O$-glucuronide, catalyzed by glycosyltransferases (uridine 5'-diphospho-glucuronosyltransferase, UDP-glucuronosyltransferase, UGT), in what is considered a detoxification route. TAM-glucuronide conjugates have been at present identified in the urine, serum, and bile of TAM-treated breast cancer patients [24].

Clinical studies suggest that the plasma concentrations of TAM and its metabolites vary widely among patients. It is likely that variable activity of the enzymes involved alter the pattern of metabolism of TAM, leading to differences in systemic exposure of TAM and/or one or more of its active metabolites [4, 11, 16, 18].

\section{TAMOXIFEN METABOLISM: IS THIS ISSUE AN ANSWER FOR TAM BENEFITS VS RISKS?}

The mechanisms underlying the variable response to tamoxifen have been the subject of intense study but remain obscure yet. It is consensual the idea that tamoxifen side effects may be related to the
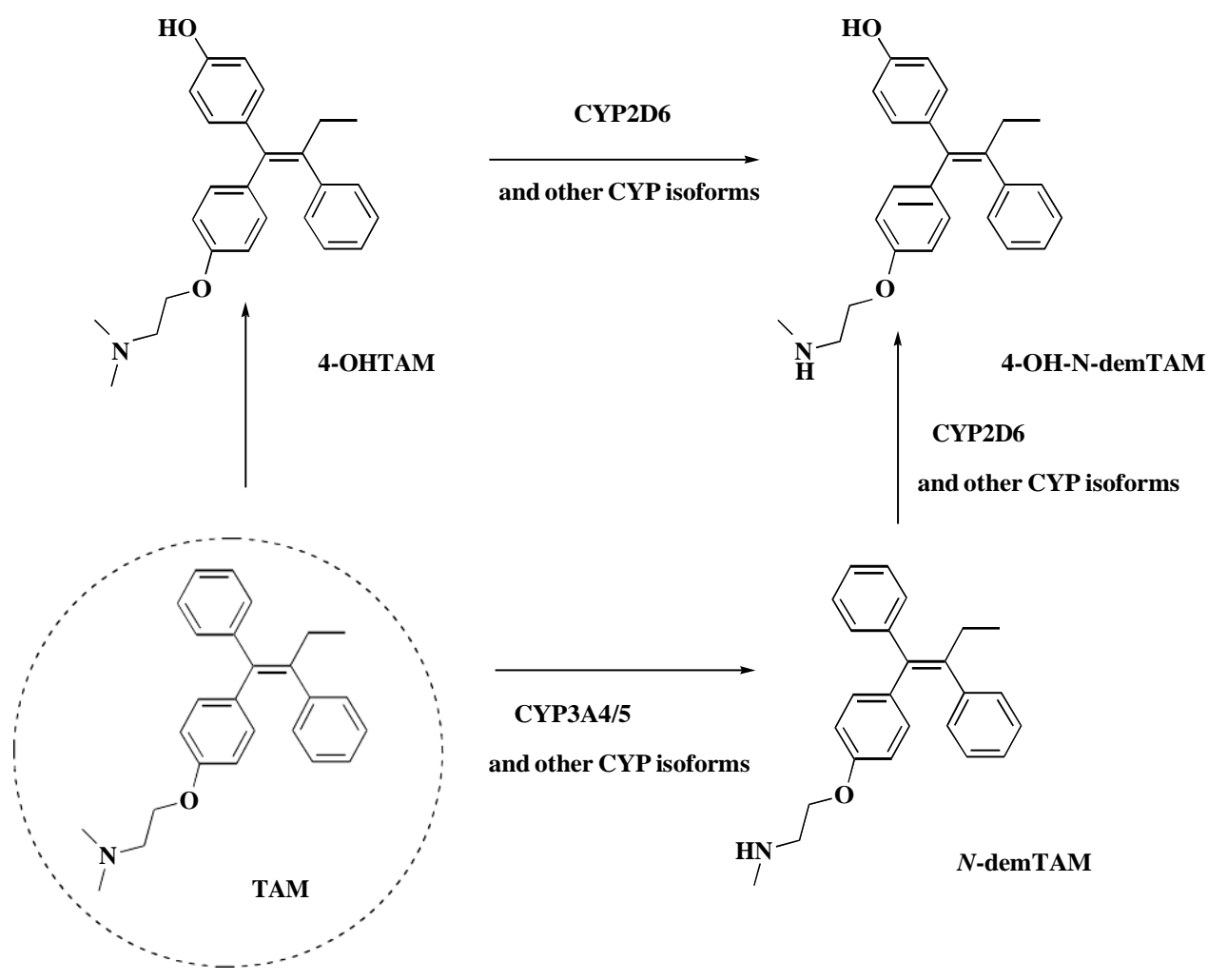

Fig. (2). Enzymatic systems engaged in the formation of the most active metabolites of tamoxifen. 

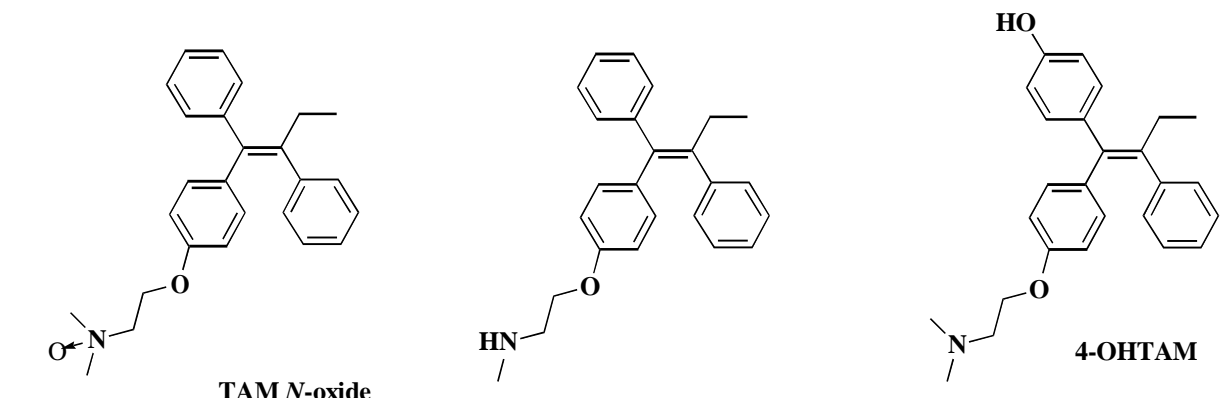

$N$-desmTAM

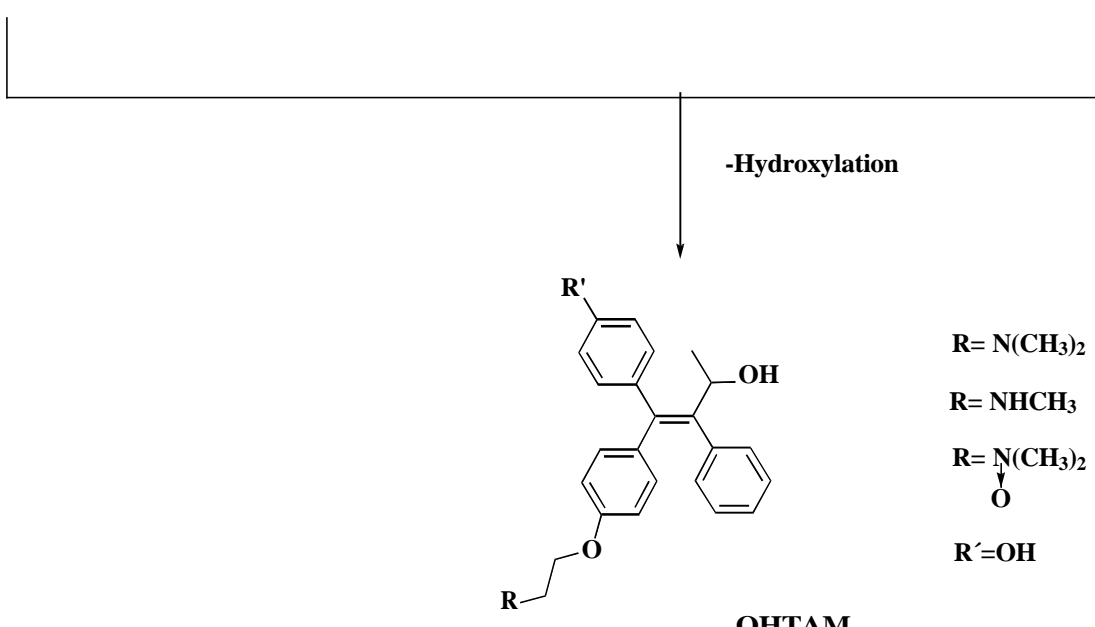

Fig. (3). Formation of -hydroxylated tamoxifen metabolites.

different patterns of metabolism. Really, the compelling evidence that tamoxifen is converted to antiestrogenic metabolites, that are more potent than TAM itself, generated one of the foremost explanatory hypothesis in which TAM metabolism is considered to be the major contribute to the interindividual variability found in clinical data $[4,5,16]$. Recent research on tamoxifen has shown that potentially a number of women with breast cancer may not receive the benefit from taking tamoxifen, because of their unique genetic make-up since they have a special version of a CYP enzyme (CYP2D6, commonly referred as 2D6) [25]. This enzyme may reduce the effectiveness of tamoxifen and increase the chance of breast cancer recurrence (Fig. 2).

On the other hand it is also believed that the cellular mechanism underlying TAM-induced carcinogenesis may be related to its partial estrogenic effect through the estrogen receptors and/or genotoxic damage induced by electrophilic metabolites. In fact, the metabolic activation of the drug, mainly done by the CYP3A family (Fig. 2 and Fig. 4), generates electrophilic species, with subsequent formation of reactive electrophilic quinines, that can interact with DNA causing damage and subsequently gene mutations (Fig. 5 and Fig. 6) [26]. However it is still controversial whether that DNA damage and the formation of TAM-DNA adducts occurs in human uterine tissues, and there is no evidence that this can be causally related to the mechanisms of carcinogenesis [5, 15]. Controversy still remains as to whether the metabolic activation result in genotoxicity or whether a non-genotoxic mechanism is responsible for the associated elevations in human endometrial carcinoma risk.

\section{ELECTROCHEMICAL OUTLOOK}

\section{General}

In drug discovery process, one crucial task is the elucidation the metabolic fate of a drug candidate in the human body [27-29]. The increasing number of new chemical entities derived in recent years requires fast screening techniques that provide both reliable and easily accessible information about the biotransformation. For economic reasons it is important to perform, these tests at an early stage to exclude unsuitable drug candidates. Therefore, several chemical and enzymatic model systems have been developed that mimic metabolic processes in vitro in order to perform a satisfactory simulation of the situation in vivo [30-32].

Electrochemistry is one of the most sensitive and versatile techniques available for the study of biomolecules [33, 34]. The use of electrochemical techniques for the determination of compounds of pharmaceutical interest in different matrices is continually gaining in importance. The inherent sensitivity and high selectivity of the techniques allow very simple determinations, both in commercial samples and in body fluids in the presence of metabolites or impurities such as precursors used in the synthesis of the compounds [3537].

Electrochemical methods are nowadays considered to be useful tools for the investigation of electron-transfer reactions and can be used for simulations of biological (i.e. metabolic) redox reactions [38-42]. In fact, a major bottleneck in metabolic profiling remains in the detection and identification of active metabolites in complex mixtures. As electrochemistry is often used for the determination of oxidation mechanisms, it can be applied as a simulation technique in drug metabolism studies [31, 43]. In fact, metabolically active sites, i.e., positions at which the drug is prone to oxidation, can thus be rapidly elucidated and possible metabolites can be directly identified.

First achievements in the use of electrochemistry as a tool for simulating the oxidative metabolism of drugs date back to the early 1980s. At that time, mechanistic comparisons between electrochemical and enzymatic oxidation reactions were of huge interest. 
A
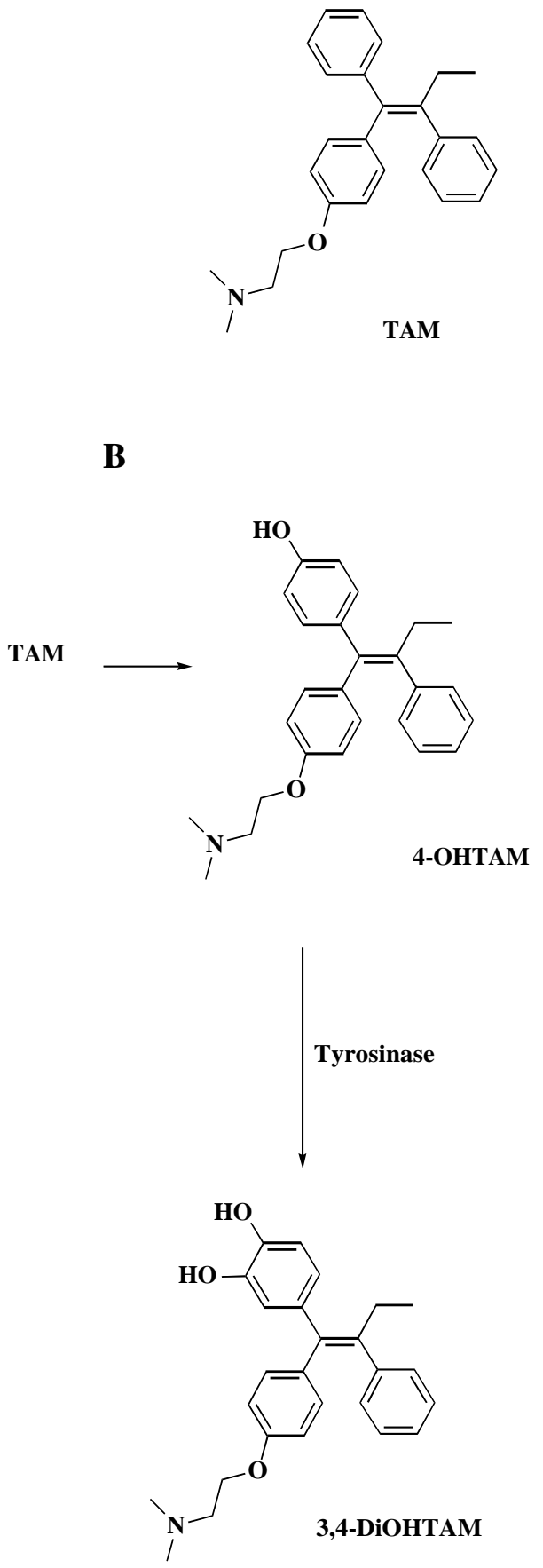

Fig. (4). Electrophilic species generated on tamoxifen metabolism.

In fact, the data on the redox properties of drugs and biomolecules might have a profound effect to understand their in vivo redox behaviour or pharmaceutical activity. Accordingly, different electrochemical studies have been addressed to mimic phase-I reactions such as aromatic hydroxylation, dehydrogenation, $\mathrm{O}-$, and $\mathrm{N}-$ dealkylation [31, 44, 45].

A special focus of drug metabolism studies is the detection of reactive metabolites which often cause severe side effects. A high proportion of drugs involved in idiosyncratic toxicity are capable of forming reactive metabolic intermediates [46-48]. The majority of these intermediates are formed after the oxidation of the parent compound to an electrophilic intermediate, which subsequently can
Oxidation

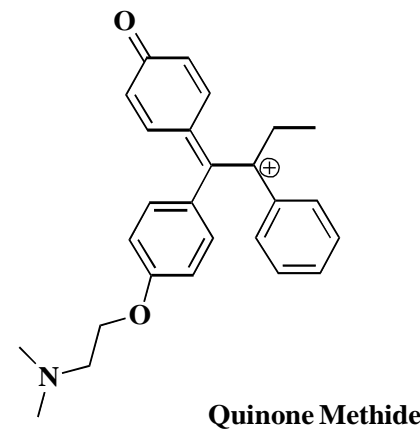

$\mathbf{R}=\mathbf{N H C H}_{3}$

$\mathbf{R}=\underset{\downarrow}{\mathbf{N}}\left(\mathrm{CH}_{3}\right)_{2}$

O

Quinone Methide

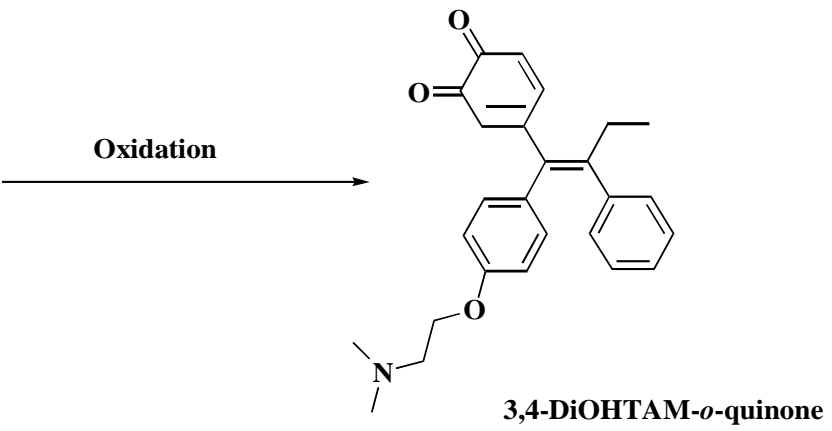

react with nucleophilic groups in cellular biomacromolecules such as lipids, proteins and DNA [48-50]. These reactive metabolites can be generated by metabolic phase-I reactions (oxidation, reduction), e.g. catalyzed by cytochrome P450s (CYP), flavin monooxygenases (FMO), and peroxidases and also by phase-II reactions (conjugation), e.g. catalyzed by UDP glucuronyltransferases (UGT) or sulfotransferases (SULT) [48].

The short lifetimes of electrophilic reactive intermediates, have boosted the development of analytical strategies mainly based in in situ $^{-}$trapping. Several approaches using "on-line metabolite generation" have been described to increase throughput in early drug metabolism studies. An instrumental approach is the use of on-line 

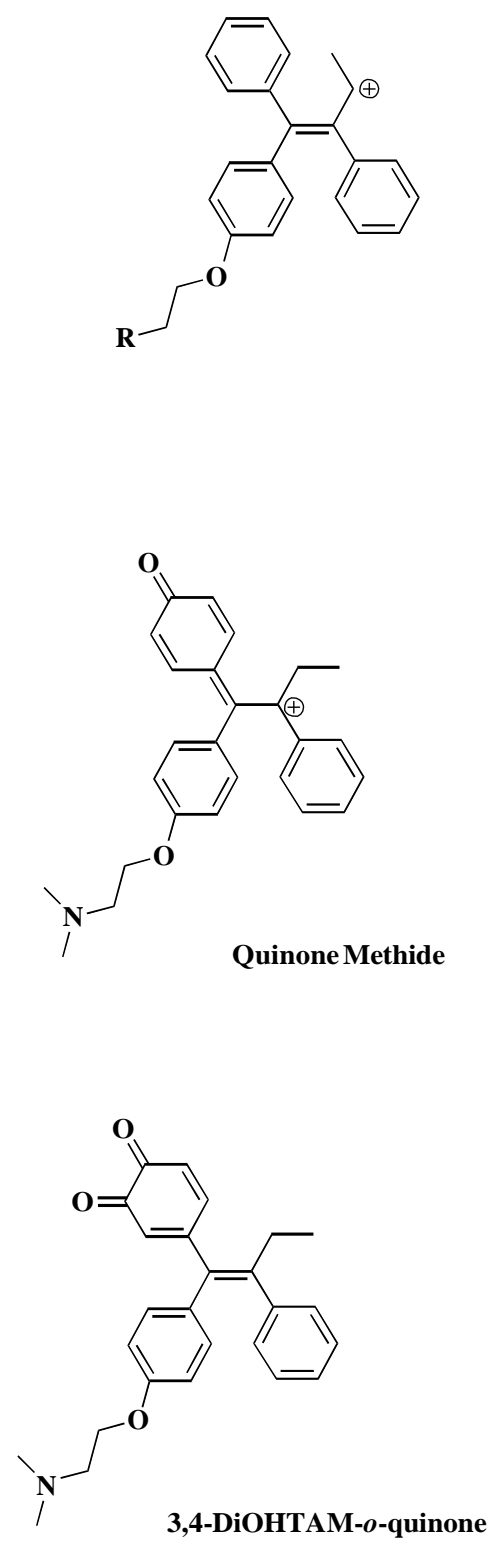

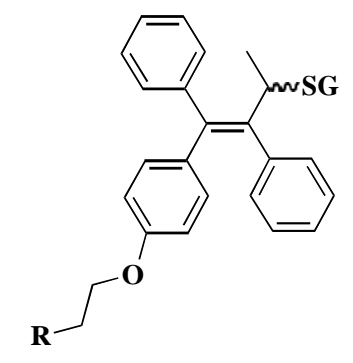

GSH
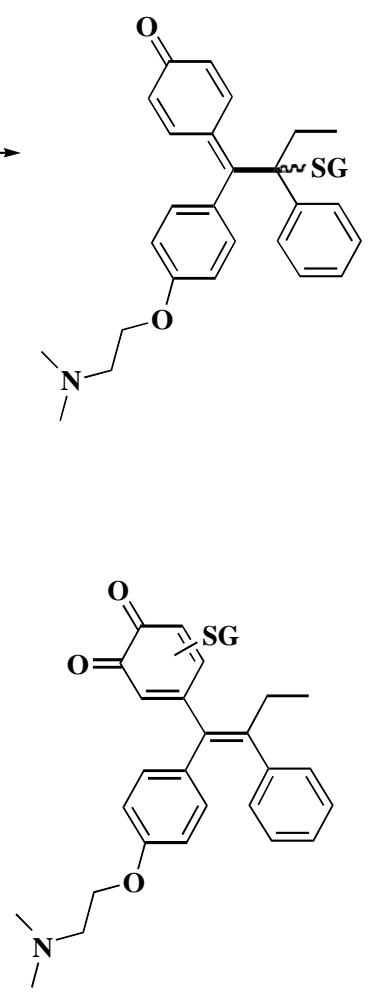

Fig. (5). Interaction of electrophilic intermediates generated during tamoxifen metabolism with gluthatione (GSH).

electrochemistry-mass spectrometry (EC-MS) to mimic cytochrome P450-catalyzed reactions [43, 51-53]. The on-line coupling of EC with LC-MS has evolved as a powerful tool for the elucidation and study of drug metabolism reactions in recent years [54-56]. Oxidative metabolism reactions are simulated electrochemically and metabolites are separated with HPLC and identified with MS. Although standard in vivo and in vitro approaches, such as the use of liver microsomes, can certainly not be replaced by EC, the purely instrumental technique has found its application in several complementary issues [56].

Damage to DNA may result in critical disturbances in the cell life and have serious impacts on the human's health. Development and improvement of analytical techniques capable of rapid and sensitive detection of various types of DNA damage and DNA damaging agents is therefore important. Electrochemical analysis of DNA offers a number of approaches in DNA damage detection as well as in sensing of DNA damaging agents in the environment, in food, in clinical samples, etc. Several reviews are available on electrochemical biosensors and particularly on applications of DNA modified electrodes as sensors for the detection of drugs or potential DNA-damaging agents [57-62].

\section{Analysis of Tamoxifen and Metabolites}

Regarding tamoxifen pharmaceutical interest as a nonsteroidal antiestrogen and despite its elevated risk of endometrial carcinoma, whether being a consequence of a DNA-damaging (genotoxic) or non-genotoxic mechanism remains obscure, the potential offered by electrochemical methods to highlight some of these uncertain issues have been poorly explored. In fact, very few studies based on electrochemistry have been performed and reported in the literature for tamoxifen and its metabolites. The majority of the data published in 


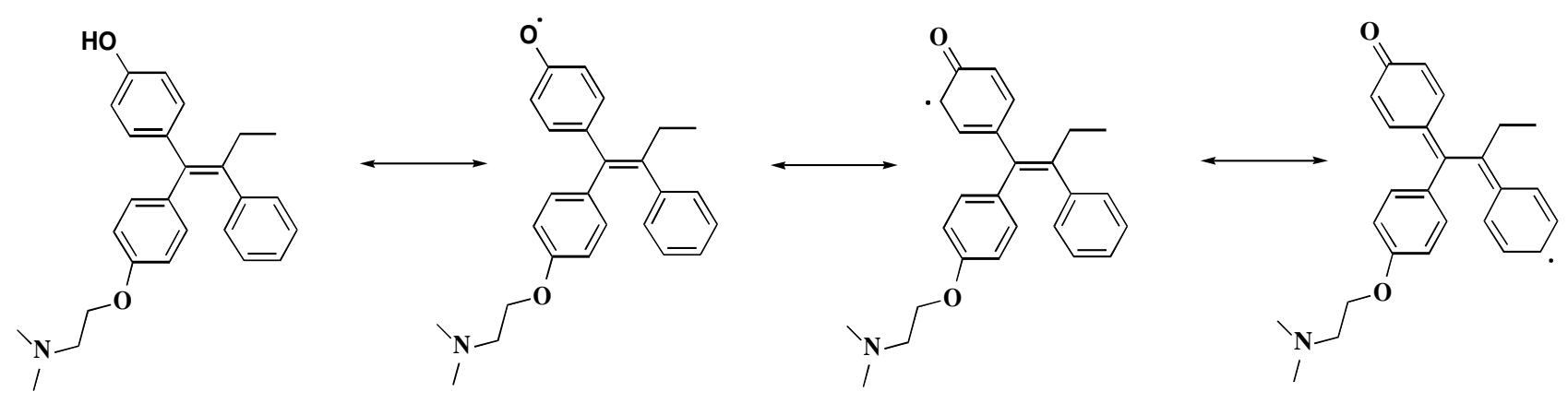

Polymerization

\section{Protein/DNA binding}

literature was attained in view of the development of simple and rapid electroanalytical methods for the determination of tamoxifen.

The polarographic and voltammetric behaviour of tamoxifen and other triphenylethene derivatives have been evaluated and the redox properties were used for its determination in pure form and in pharmaceutical tablets [63]. The observed polarographic reduction of tamoxifen was assigned to the cleavage of the double ethylenic bond involving the transfer of two electron and two protons per molecule of reactant (Fig. 8). On the other hand, the anodic signal was attributed to the cyclization reaction to form the corresponding phenanthrene derivative (Fig. 8). A similar mechanism was proposed for the oxidation of tamoxifen at various electrodes [64, 65].

Although the data found in literature concerning the oxidative mechanism of tamoxifen is coincident, and follows the generally accepted mechanism proposed for phenylethenes derivatives oxidation $[66,67]$ there are some remaining issues that need to be pursued. In fact, in addition to the double ethylenic bond, tamoxifen's molecular structure includes other functional groups that could also undergo oxidative reactions (Fig. 1), namely an oxygen aromatic ether and an aliphatic tertiary amine. Compounds containing these organic moieties have been described in literature as being electroactive and presenting anodic waves at potentials similar to that found for tamoxifen (ca. 1.0 V) [68-71]. Furthermore, as mentioned previously tamoxifen metabolism in humans comprises mainly $\mathrm{N}$ desalkylation of the aminoethoxy side chain. The major oxidative metabolite of tamoxifen found in human plasma is $N$-desmethyl tamoxifen ( $N$-demTAM).

The oxidation of tamoxifen at a glassy carbon flow detector was exploited for its amperometric chromatographic monitoring in cell culture medium [72]. The absolute detection limit obtained was 100 pg for tamoxifen at a sensitivity of $1 \mathrm{nA} / \mathrm{V}$.

A highly sensitive adsorptive chronopotentiometric stripping voltammetric method was proposed for the analysis of trace tamoxifen at a glassy carbon electrode [64]. The adsorptive stripping response was evaluated with respect to different variables and the developed method was successfully used for tamoxifen determination in urine, with a detection limit of $410^{-10} \mathrm{M}$ after 

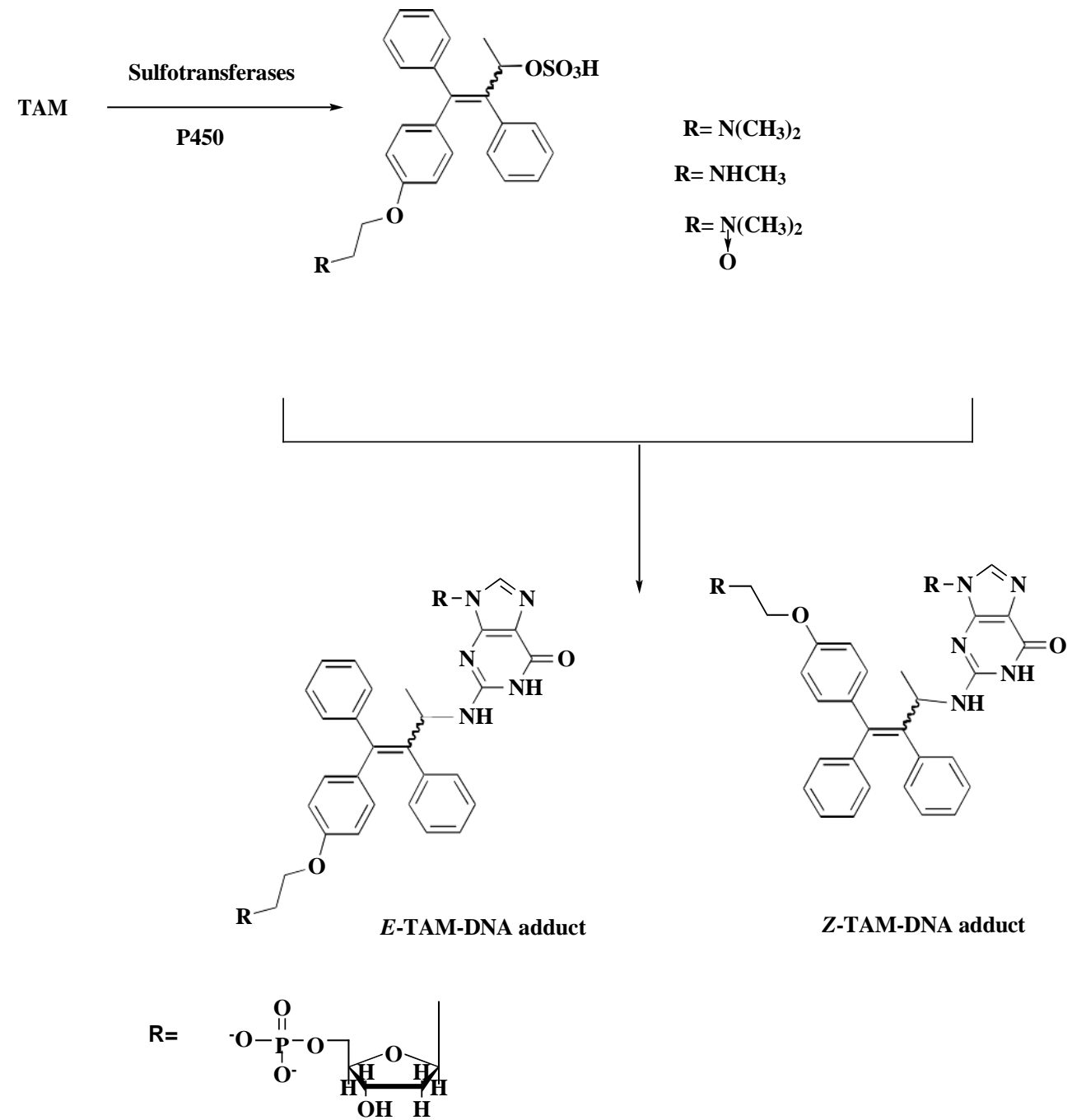

Fig. (7). Proposed pathway involved in the formation of tamoxifen-DNA adducts.

4 min preconcentration.

A single-sweep voltammetric method was proposed for the determination of tamoxifen [65] in pharmaceutical dosage samples. The described methodology took advantage of both the higher capacity for accumulation of carbon paste electrode toward tamoxifen and the rapidity of single-sweep voltammetry. The proposed method, characterized by its rapidity and higher sensitivity, was evaluated by analyzing tamoxifen citrate tablets, and yielded a detection limit of $1.010^{-10} \mathrm{M}$ without any preconcentration.

Recently, an ultrasensitive flow-injection electrochemical method was proposed for the determination of tamoxifen in urine, plasma and pharmaceutical preparations [73]. The reported methodology describes a novel, sensitive and widely applicable fast Fourier transform (FFT) square wave voltammetry (SWV) using a gold microelectrode in flow-injection system. FFT-SWV was demonstrated to provide sensitive detection based on oxidation of the electrode surface. Under optimum conditions at flow-injection system, a detection limit of 3.0 $10^{12} \mathrm{M}$ was obtained for tamoxifen.

\section{Future Prospects}

Tamoxifen remains a frontline treatment for hormone-responsive breast cancer despite its use being associated with an elevated risk of developing endometrial carcinoma. The mechanisms underlying the induction of endometrial tumors by tamoxifen have yet to be established, although both the genotoxic and the hormonal properties of the drug have been implicated.

As many drugs, tamoxifen is biotransformed to pharmacologically active metabolites that can significantly contribute to the overall pharmacological or adverse effects of the drug. For the most part, the pharmacological actions attributed to active metabolites are similar to those of the parent. However, active metabolites could also mediate therapeutic effects that are synergistic or even the reverse of the pharmacological action of the parent drug. Therefore, to fully understand the mechanism of action of a drug, it is imperative to recognize the role of active metabolites.

Recently, researchers have utilized an electrochemistry-based approach to answer many questions about a compound's metabolic behaviour [31, 43, 52, 53]. Electrochemical systems take advantage of the relationship between oxidation/reduction chemistry and CYP450-mediated oxidative drug metabolism (Phase I metabolism) to provide a wide variety of information on metabolic characteristics. Even though it is still not possible to simulate the complete spectrum of CYP mediated metabolic reactions adequately, and differences between the electrochemically and enzymatically generated metabolites occur, this technique has already shown its potential as a tool that is complementary to existing techniques [31, $43,53]$. 

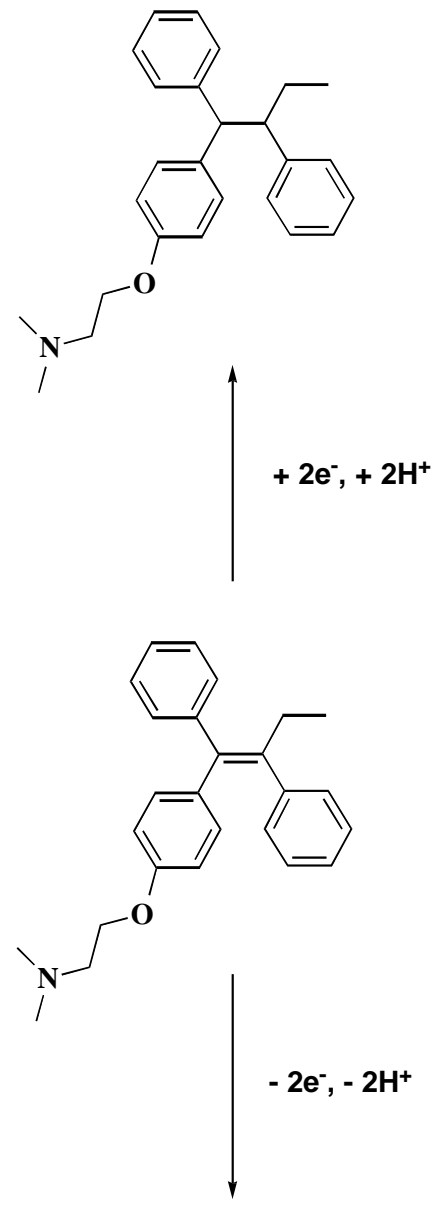

TAM

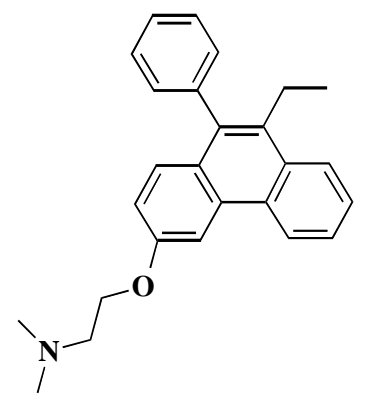

Fig. (8). Proposed mechanism for the electrochemical reduction and oxidation of tamoxifen.

Moreover, the toxicity of a number of drugs has been often associated with their ability to form reactive metabolic intermediates by oxidation of the parent compound to an electrophilic intermediate, which subsequently can react with nucleophilic functional groups in cellular biomacromolecules, such as DNA. During the past decades, electrochemistry has been shown as a powerful tool in studies of nucleic acid structure, interactions and damage.

The improvement of quality of life has stimulated considerable research in drug design bioavailability and safety. Thus, in order to achieve these targets, highly sensitive and specific methods of analysis are necessary. Modern electrochemical methods advantages include high sensitivity, wide applicability and low-cost equipment. Besides all these potentialities electrochemical methods have been scarcely used to comprehend tamoxifen's biotransformation and its DNA damaging mechanisms [74].
The development of new detection methods based on electrochemistry are particularly interesting because their sensitivity and the opportunity to miniaturize the technology $[75,76]$. The attractive properties of electrochemical devices are extremely promising for improving the efficiency of diagnostic testing and therapy monitoring of tamoxifen. Nevertheless, some new studies on the voltammetric behaviour of tamoxifen should be accomplished in order to clarify its oxidative mechanism.

The development of electrochemical DNA-biosensors for the study of tamoxifen could open a wide perspective using particularly sensitive and selective methods for the detection of specific interactions. This biosensor would enable pre-concentration of tamoxifen onto the sensor surface and in situ electrochemical generation of radical intermediates, which can cause damage to the DNA immobilized on the electrode surface and can be detected electrochemically. Voltammetric methods can therefore contribute to elucidation of the mechanism through which DNA is oxidatively damaged by tamoxifen and its metabolites.

\section{REFERENCES}

[1] Rossi, A.; Colantuoni, G.; Maione, P.; Ferrara, C.; Airoma, G.; Barzelloni, M.L.; Castaldo; V.; Gridelli, C. Chemotherapy of breast cancer in the elderly. Curr. Med. Chem., 2005, 12, 297-310.

[2] Musa, M.A.; Khan, M.O.F.; Cooperwood, J.S. Medicinal chemistry and emerging strategies applied to the development of selective estrogen receptor modulators (SERMs). Curr. Med. Chem., 2007, 14, 1249-1261.

[3] Powles, T.J. Efficacy of tamoxifen as treatment of breast cancer. Semin. Oncol., 1997, 24 (Suppl. 1), 48-54.

[4] MacGregor, J.I.; Jordan, V.C. Basic guide to the mechanisms of antiestrogen action. Pharmacol. Rev., 1998, 50 (2), 151-196 and references herein.

[5] Singh, M.N.; Stringfellow, H.F.; Paraskevaidis, E.; Martin-Hirsch, P.L.; Martin, F.L. Tamoxifen: important considerations of a multifunctional compound with organ-specific properties. Cancer Treat. Rev., 2007, 33, 91-100.

[6] Baum, M. Tamoxifen - the treatment of choice. Why look for alternatives? Br. J. Cancer, 1998, 78 (Suppl. 4), 1-4.

[7] Goss, P.E.; Ingle, J.N.; Martino, S.; Robert, N.J.; Muss, H.B.; Piccart, M.J.; Castiglione, M.; Tu, D.; Shepherd, L.E.; Pritchard, K.I.; Livingston, R.B.; Davidson, N.E.; Norton, L.; Perez, E.A.; Abrams, J.S.; Cameron, D.A.; Palmer, M.J.; Pater, J.L. Randomized trial of letrozole following tamoxifen as extended adjuvant therapy in receptor-positive breast cancer: updated findings from NCIC CTG MA.17. J. Natl. Cancer Inst., 2005, 97, 1262-1271.

[8] Fisher, B.; Costantino, J.P.; Wickerham, D.L.; Redmond, C.K.; Kavanah, M.; Cronin, W.M.; Vogel, V.; Robidoux, A.; Dimitrov, N.; Atkins, J.; Daly, M.; Wieand, S.; Tan-Chiu, E.; Ford, L.; Wolmark, N. Tamoxifen for the prevention of breast cancer: report of the national surgical adjuvant breast and bowel project $\mathrm{P}-1$ study. $J$. Natl. Cancer Inst., 1998, 90, 1371-1388.

[9] Cuzick, J.; Powles, T.; Veronesi, U.; Forbes, J.; Edwards, R.; Ashley, S.; Boyle, P. Overview of the main outcomes in breast-cancer prevention trials. Lancet, 2003, 361, 296-300.

[10] Smith, L.L; Brown, K.; Carthew, P.; Lim, C.-K.; Martin, E.A.; Styles, J.; White, I.N.H. Chemoprevention of breast cancer by tamoxifen: risks and opportunities. Crit. Rev. Toxicol., 2000, 30, 571-594.

[11] Brown, K. Is tamoxifen a genotoxic carcinogen in women? Mutagenesis, 2009, 24, 391-404.

[12] Turken, S.; Siris, E.; Seldin, D.; Flaster, E.; Hyman, G.; Lindsay, R. Effects of tamoxifen on spinal bone density in women with breast cancer. J. Natl. Cancer Inst., 1989, 81, 1086-1088.

[13] Gylling, H.; Pyrhonen, S.; Mantyla, E.; Maenpaa, H.; Kangas, L.; Miettinen, T. A. Tamoxifen and toremifene lower serum cholesterol by inhibition of delta 8-cholestenol conversion to lathosterol in women with breast cancer. J. Clin. Oncol., 1995, 13, 2900-2905.

[14] Ruenitz, P.C. Drugs for osteoporosis prevention: mechanisms of bone maintenance. Curr. Med. Chem., 1995, 2, 791-802.

[15] Badia, E.; Oliva, J.; Balaguer, P.; Cavaillès, V. Tamoxifen resistance and epigenetic modifications in breast cancer cell lines. Curr. Med. Chem., 2007, 14, 3035-3043. 
[16] White, I.N.H. Tamoxifen: is it safe? Comparison of activation and detoxication mechanisms in rodents and in humans. Curr. Drug Metab., 2003, 4, 223-239 and references herein.

[17] Desta, Z.; Ward, B.A.; Soukhova, N.V.; Flockhart, D.A. Comprehensive evaluation of tamoxifen sequential biotransformation by the human cytochrome P450 system in vitro: prominent roles for CYP3A and CYP2D6. J. Pharmacol. Exp. Ther., 2004, 310, 10621075.

[18] Kim, S.Y.; Laxmi, Y.R.S.; Suzuki, N.; Ogura, K.; Watabe, T.; Duffel, M.W.; Shibutani, S. Formation of tamoxifen-DNA adducts via $o$-sulfonation, not $o$-acetylation, of -hydroxytamoxifen in rat and human livers. Drug Metab. Dispos., 2005, 33, 1673-1678 and references herein.

[19] Chen, G.; Yin, S.; Maiti, S.; Shao, X. 4-Hydroxytamoxifen sulfation metabolism. J. Biochem. Mol. Toxicol., 2002, 16, 279-285.

[20] Zhang, F.; Fan, P.W.; Liu, X.; Shen, L.; van Breemen, R.B.; Bolton, J.L. Synthesis and reactivity of a potential carcinogenic metabolite of tamoxifen: 3,4-dihydroxytamoxifen-o-quinone. Chem. Res. Toxicol., 2000, 13, 53-62.

[21] Kuramochi, H. Conformational studies and electronic structures of tamoxifen and toremifene and their allylic carbocations proposed as reactive intermediates leading to DNA adduct formation. J. Med. Chem., 1996, 39, 2877-2886.

[22] Costa, G.G.; Marques, M.M.; Beland, F.A.; Freeman, J.P.; Churchwell, M.I.; Doerge, D.R. Quantification of tamoxifen DNA adducts using on-line sample preparation and HPLC-electrospray ionization tandem mass spectrometry. Chem. Res. Toxicol., 2003, 16, 357-366.

[23] Kim, S.Y.; Suzuki, N.; Laxmi, Y.R.S.; Shibutani, S. Inefficient repair of tamoxifen-DNA adducts in rats and mice. Drug Metab. Dispos., 2006, 34, 311-317.

[24] Zheng, Y.; Sun, D.; Sharma, A.K.; Chen, G.; Amin, S.; Lazarus, P. Elimination of antiestrogenic effects of active tamoxifen metabolites by glucuronidation. Drug Metab. Dispos., 2007, 35, 19421948.

[25] Ferraldeschi, R.; Newman, W.G. The impact of CYP2D6 genotyping on tamoxifen treatment. Pharmaceuticals, 2010,3, 1122-1138.

[26] Wegman, P.; Vainikka, L.; Stål, O.; Nordenskjöld, B.; Skoog, L.; Rutqvist, L.-E.; Wingren, S. Genotype of metabolic enzymes and the benefit of tamoxifen in postmenopausal breast cancer patients. Breast Cancer Res., 2005, 7, R284-R290.

[27] Kumar, G.N.; Surapaneni, S. Role of drug metabolism in drug discovery and development. Med. Res. Rev., 2001, 21, 397-411.

[28] Fura, A.; Shu, Y.-Z.; Zhu, M.; Hanson, R.L.; Roongta, V.; Humphreys, W.G. Discovering drugs through biological transformation: role of pharmacologically active metabolites in drug discovery. $J$. Med. Chem., 2004, 47, 4339-4351.

[29] Fura, A. Role of pharmacologically active metabolites in drug discovery and development. Drug Discov. Today, 2006, 11, 133142.

[30] Chen, Y.; Monshouwer, M.; Fitch, W.L. Analytical tools and approaches for metabolite identification in early drug discovery. Pharm. Res., 2007, 24, 248-257.

[31] Lohmann, W.; Karst, U. Biomimetic modeling of oxidative drug metabolism. Strategies, advantages and limitations. Anal. Bioanal. Chem., 2008, 391, 79-96.

[32] Shu, Y.-Z.; Johnson, B.M.; Yang, T.J. Role of biotransformation studies in minimizing metabolism-related liabilities in drug discovery. AAPS J., 2008, 10, 178-192.

[33] Kissinger, P.T.; Heineman, W.R. Laboratory Techniques in Electroanalytical Chemistry, Marcel Dekker: New York, 1996.

[34] Brajter-Toth, A.; Chambers, J.Q. Electroanalytical Methods for Biological Materials, Marcel Dekker: New York, 2002.

[35] Özkan, S.A.; Uslu, B.; Aboul-Enein, H.Y. Analysis of pharmaceuticals and biological fluids using modern electroanalytical techniques. Crit. Rev. Anal. Chem., 2003, 33, 155-181.

[36] Milhazes, N.; Martins, P.; Uriarte, E.; Garrido, J.; Calheiros, R.; Marques, M.P.M.; Borges, F. Electrochemical and spectroscopic characterisation of amphetamine-like drugs: application to the screening of 3,4-methylenedioxymethamphetamine (MDMA) and its synthetic precursors. Anal. Chim. Acta, 2007, 596, 231-241.

[37] Radi, A.-E. Recent updates of chemically modified electrodes in pharmaceutical analysis. Comb. Chem. High Throughput Screen., 2010, 13, 728-752.

[38] Eckert, G.M.; Gutmann, F.; Keyzler, H. Electropharmacology, CRC Press: Boca Raton, 1990
[39] Dryhurst, G.; Kadish, K.M.; Shceller, F.; Renneberg, R. Biological Electrochemistry, Academic Press: New York, 1982.

[40] Berry, M.N. An electrochemical interpretation of metabolism. FEBS Lett., 1981, 134, 133-138.

[41] Abreu, F.C.; Ferraz, P.A.L.; Goulart, M.O.F. Some applications of electrochemistry in biomedical chemistry. Emphasis on the correlation of electrochemical and bioactive properties. J. Braz. Chem. Soc., 2002, 13, 19-35.

[42] Meyer, D.F.; Gamache, P.H.; Acworth, I.N. In: Metabolome Analyses: Strategies for Systems Biology, Vaidyanathan, S.; Harrigan, G.G.; Goodacre, R., Eds.; Springer: New York, 2005; Ch. 8, pp. 119-135.

[43] Karst, U. Electrochemistry/mass spectrometry (EC/MS) - A new tool to study drug metabolism and reaction mechanisms. Angew. Chem. Int. Ed., 2004, 43, 2476-2478.

[44] Deng, H.T.; Van Berkel, G.J. A thin-layer electrochemical flow cell coupled on-line with electrospray-mass spectrometry for the study of biological redox reactions. Electroanalysis, 1999, 11, 857865.

[45] Johansson, T.; Weidolf, L.; Jurva, U. Mimicry of phase I drug metabolism - novel methods for metabolite characterization and synthesis. Rapid Commun. Mass Spectrom., 2007, 21, 2323-2331.

[46] Korfmacher, W.A., Ed. Using mass spectrometry for drug metabolism studies, CRC Press: Boca Raton, Florida, 2005.

[47] Staack, R.F.; Hopfgartner, G. New analytical strategies in studying drug metabolism. Anal. Bioanal. Chem. 2007, 388, 1365-1380.

[48] Kalgutkar, A.S.; Gardner, I.; Obach, R.S.; Shaffer, C.L.; Callegari, E.; Henne, K.R.; Mutlib, A.E.; Dalvie, D.K.; Lee, J.S.; Nakai, Y.; O’Donnell, J.P.; Boer, J.; Harriman, S.P. A comprehensive listing of bioactivation pathways of organic functional groups. Curr. Drug Metab., 2005, 6, 161-225.

[49] Walgren, J.L.; Mitchell, M.D.; Thompson, D.C. Role of metabolism in drug-induced idiosyncratic hepatotoxicity. Crit. Rev. Toxicol., 2005, 35, 325-361.

[50] Liebler, D.C.; Guengerich, F.P. Elucidating mechanisms of druginduced toxicity. Nat. Rev. Drug Discov., 2005, 4, 410-420.

[51] Jurva, U.; Wikström, H. V.; Bruins, A. P. In vitro mimicry of metabolic oxidation reactions by electrochemistry/mass spectrometry. Rapid Commun. Mass Spectrom., 2000, 14, 529-533.

[52] Permentier, H.P.; Bruins, A.P.; Bischoff, R. Electrochemistry-Mass spectrometry in drug metabolism and protein research. Mini Rev. Med. Chem., 2008, 8, 46-56.

[53] Álvarez-Lueje, A.; Dragnic, S. B. Stability and drug metabolism assessed by electrochemical methods. Comb. Chem. High Throughput Screen., 2010, 13, 712-727.

[54] Lohmann, W.; Karst, U. Electrochemistry meets enzymes: instrumental on-line simulation of oxidative and conjugative metabolism reactions of toremifene. Anal. Bioanal. Chem., 2009, 394, 13411348.

[55] Odijk, M.; Baumann, A.; Lohmann, W.; van den Brink, F. T. G.; Olthuis, W.; Karst, U.; van den Berg, A. A microfluidic chip for electrochemical conversions in drug metabolism studies. Lab Chip, 2009, 9, 1687-1693.

[56] Lohmann, W.; Baumann, A; Karst, U. Electrochemistry and LCMS for metabolite generation and identification: tools, technologies and trends. LC GC Eur., 2010, 23, 8-16.

[57] Fojta, M. Electrochemical sensors for DNA interactions and damage. Electroanalysis, 2002, 14, 1449-1463.

[58] Palecek, E.; Fojta, M.; Jelen, F.; Vetterl, V. In: The Encyclopedia of Electrochemistry, Bard, A.J.; Stratsmann, M., Eds.; Wiley-VCH: Weinheim, 2002; Vol. 9, pp. 365-429.

[59] Kerman, K.; Kobayashi, M.; Tamiya, E. Recent trends in electrochemical DNA biosensor technology. Meas. Sci. Technol., 2004, $15, \mathrm{R} 1-\mathrm{R} 11$.

[60] Oliveira-Brett, A.M. In: Encyclopedia of Sensors, Grimes, C.A.; Dickey, E.C.; Pishko, M.V., Eds.; American Scientific Publishers: California, 2006; Vol. 3, pp. 301-314.

[61] Oliveira Brett, A.M.; Diculescu, V.; Chiorcea-Paquim, A.M.; Serrano. S.H.P. In: Comprehensive Analytical Chemistry (CAC), Alegret, S.; Merkoçi, A., Eds.; Elsevier: Amsterdam, 2007, Vol. 49, pp. 413-437.

[62] Oliveira-Brett, A.M. In: Bioelectrochemistry: Fundamentals, Experimental Techniques and Applications, Bartlett, P.N., Ed.; John Wiley: Chichester, 2008, pp. 411-442. 
[63] Fijalek, Z.; Chodkowski, J.; Warowna, M. Polarographic studies of drugs of triphenylethene derivatives. J. Electroanal. Chem., 1987, 226, 129-136.

[64] Wang, J.; Cai, X.; Fernandes, J.R.; Ozsoz, M.; Grant, D.H. Adsorptive potentiometric stripping analysis of trace tamoxifen at a glassy carbon electrode. Talanta, 1997, 45, 273-278.

[65] Guo, X.-X.; Song, Z.-J.; Tian, X.-J.; Song, J.-F. Single-sweep voltammetric determination of tamoxifen at carbon paste electrode. Anal. Lett., 2008, 41, 1225-1235.

[66] Stuart, J.D.; Ohnesorge, W.E. Electrochemical oxidation of phenylethylenes. I. Conversion of tetraphenylethylene to 9,10diphenylphenanthrene. J. Am. Chem. Soc., 1971, 93, 4531-4536.

[67] Schreivogel, A.; Maurer, J.; Winter, R.; Baro, A.; Laschat, S. Synthesis and electrochemical properties of tetrasubstituted tetraphenylethenes. Eur. J. Org. Chem., 2006, 3395-3404.

[68] Lund, H.; Baiser, M. M., Ed. Organic Electrochemistry, 3rd ed.; Marcel Dekker: New York, 1991.

[69] Garrido, J.M.P.J.; Delerue-Matos, C.; Borges, F.; Macedo, T.R.A.; Oliveira-Brett, A.M. New insights into the oxidation pathways of apomorphine. J. Chem. Soc., Perkin Trans., 2002, 2, 1713-1717.

[70] Garrido, E.M.P.J.; Garrido, J.M.P.J.; Esteves, M.; Santos-Silva, A.; Marques, M.P.M.; Borges, F. Voltammetric and DFT studies on viloxazine: analytical application to pharmaceuticals and biological fluids. Electroanalysis, 2008, 20, 1454-1462.

[71] Garrido, E.M.; Garrido, J.; Calheiros, R.; Marques, M.P.M.; Borges, F. Fluoxetine and norfluoxetine revisited: new insights into the electrochemical and spectroscopic properties. J. Phys. Chem. A., 2009, 113, 9934-9944.

[72] Chamart, S.; Hanocq, M.; Helson, M.; Devleeschouwer, N.; Leclercq, G. Determination of 2-methyl derivatives of tamoxifen in cell culture medium using high-performance liquid chromatography and electrochemical detection. J. Chromatogr. B., 1989, 496, 365-375.

[73] Daneshgar, P.; Norouzi, P.; Ganjali, M.R.; Zamani, H.A. Ultrasensitive flow-injection electrochemical method for detection of anticancer drug tamoxifen. Talanta, 2009, 77, 1075-1080.

[74] Zhao, L.; Krishnan, S.; Zhang, Y.; Schenkman, J.B.; Rusling, J.F. Differences in metabolite-mediated toxicity of tamoxifen in rodents versus humans elucidated with DNA/microsome electro-optical arrays and nanoreactors. Chem. Res. Toxicol., 2009, 22, 341-347.

[75] Wang, J. Electrochemical biosensors: towards point of care cancer diagnostics. Biosens. Bioelectron., 2006, 21, 1887-1892.

[76] Tothill, I.E. Biosensors for cancer markers diagnosis. Semin. Cell Dev. Biol., 2009, 20, 55-62. 